\title{
Urgences
}

\section{Chaleureuse (illustrations de David Carles)}

\section{Vianney Gallant}

Numéro 8, 4e trimestre 1983

Littérature jeunesse

URI : https://id.erudit.org/iderudit/025124ar

DOI : https://doi.org/10.7202/025124ar

Aller au sommaire du numéro

Éditeur(s)

Urgences

ISSN

0226-9554 (imprimé)

1927-3924 (numérique)

Découvrir la revue

Citer ce document

Gallant, V. (1983). Chaleureuse (illustrations de David Carles). Urgences, (8),

91-102. https://doi.org/10.7202/025124ar d'utilisation que vous pouvez consulter en ligne.

https://apropos.erudit.org/fr/usagers/politique-dutilisation/ 


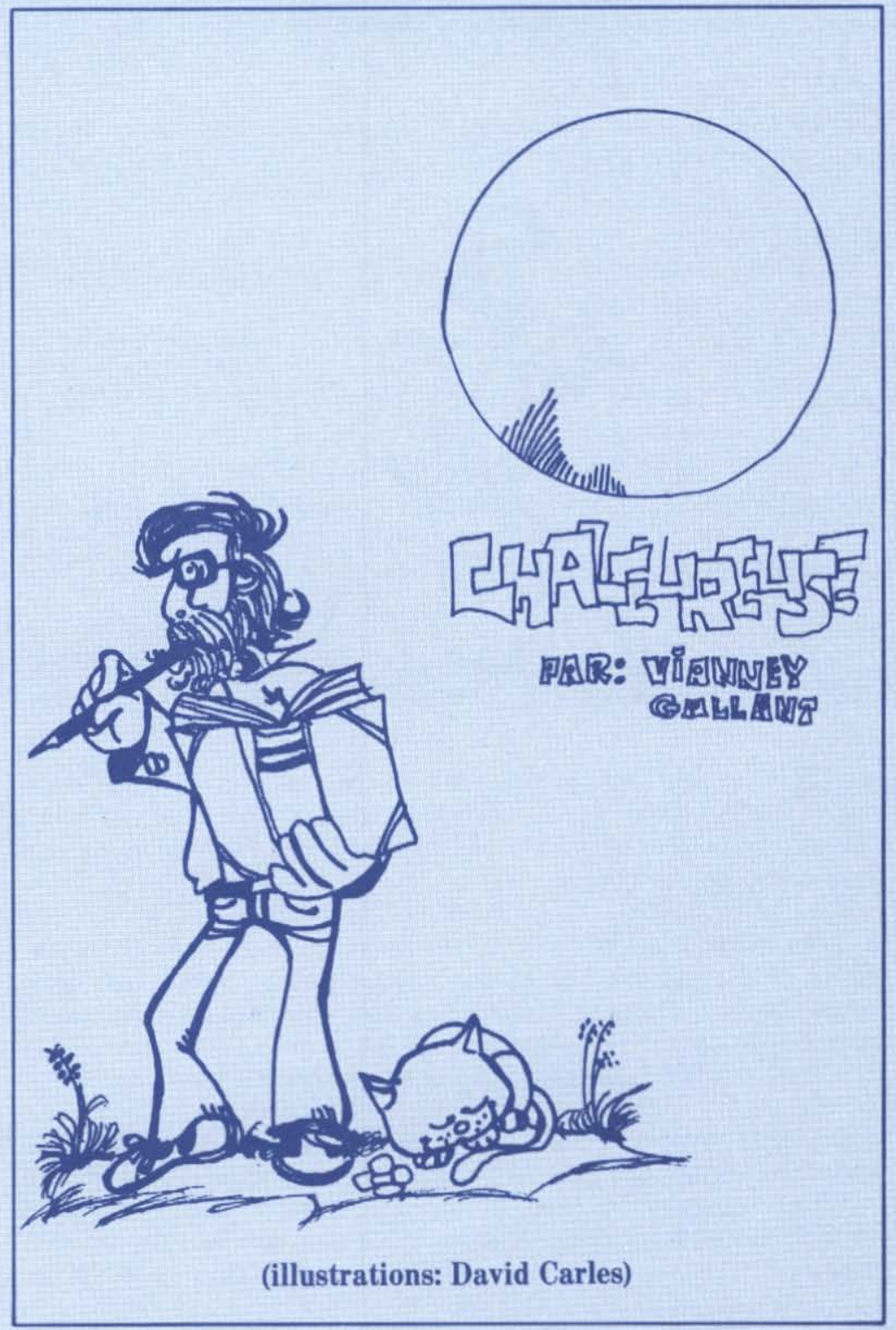




\section{CHALEUREUSE*}

De l'auteur: je prête ma plume à ma petite chatte, pour qu'elle vous raconte son histoire.

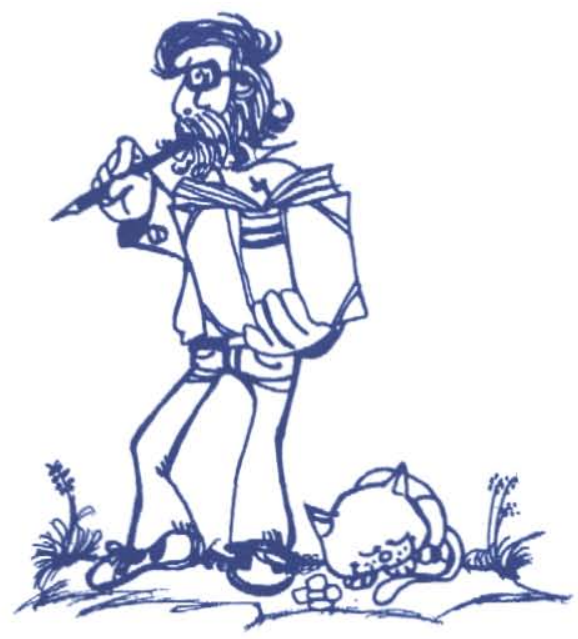

Je m'appelle chaleureuse. Je suis née dans un rang de SaintJovite, petit village des Laurentides situé à environ cent vingt kilomètres de Montréal. C'était un matin du mois de mars: un beau matin de printemps.

Ma mère Charlotte m'a portée soixante-deux jours dans son ventre. Puis, elle me rendit au jour en même temps que trois frères chatons. Dans la maisonnée, trois mamans chattes eurent leurs bébés la même semaine: ce fut une grande fête. Nous étions dix petits à nous disputer les tétines de nos mamans.

D'un commun accord, elles avaient décidé de partager la tâche de l'allaitement et d'accueillir n'importe lequel des petits qui viendrait satisfaire sa faim et sa soif, dans le creux de leurs ventres. Comme ils étaient doux et chauds les ventres de nos mamans!

- Adaptation du conte Chaleureuse de Vianney Gallant. 
Notre papa, lui, contemplait sa progéniture d'un oeil à la fois fier et indifférent. II était noir comme la nuit et ses yeux perçants chassaient les ombres et les souris. C'était un chat noir, mais jamais il n'avait porté malchance.

La maison, qui nous servait d'abri, appartenait à un couple d'êtres humains, un peu retirés dans un coin de campagne. En face de notre demeure, à dix mètres de la maison, un ruisseau gonflé par l'eau de la fonte des neiges, chantait la saison des recommencements. II y avaient beaucoup d'arbres autour de la maison. Je sais que nos mamans et notre papa y ont joué maintes fois, au chat et à la souris.

La maison était chauffée au bois et nous sommeillions tous les dix, dans notre litière, en savourant les bonnes odeurs qui émanaient du poêle. Après chaque tétée, nos mamans nous léchaient partout pour faire notre toilette. Ça chatouillait et c'était un peu rude; mais après ce nettoyage, nous étions tous bien propres. Elles faisaient ça deux fois par jour.

Dix jours après ma naissance, j'ouvris les yeux pour la première fois. Ce fut une grande joie pour moi de découvrir l'univers qui m'entourait.

Deux de nos mamans étaient tachetées de brun et de blanc; quatre petits chatons leur ressemblaient beaucoup. Trois autres petits frères avaient le poil gris, rayé de blanc; ils étaient le vrai portrait de leur mère Charlotte. Les deux derniers arrivés de notre petite communauté étaient complètement noirs comme notre papa.

De toute la bande, j'étais la seule de ma couleur: entre le gris et le noir, vous savez, comme la couleur du soir, quelques instants seulement avant que la nuit s'installe jusqu'au matin. J'étais ronde comme un petit ourson, et mon duvet de poil était doux comme la couverture de laine qui servait de matelas à notre litière. 
Après deux semaines de tétée et de sommeil constant, je commençai peu à peu à aimer les jeux que nous faisions, mes frères et moi. J'étais de taille à me mesurer avec tous: à la course, comme à nos petits chamaillages de bébés chats. D'un coup de patte, je pouvais envoyer rouler un autre sur le dos sans que pour cela il m'en garde rancune.

Le soir, après le souper, nos mères se couchaient sur le plancher et faisaient semblant de dormir. Elles bougeaient la queue pour que nous exercions nos réflexes en tentant d'immobiliser celle-ci avec nos pattes encore toutes menues. $C^{\prime}$ était une belle vie de petit chat.

Un jour, alors que je commençais à peine à comprendre le langage de mes parents, je surpris cette grave conversation:

- Miaou! Je crois que nous n'en avons plus pour longtemps à vivre tous ici, dit tante Minette.

- Miaou! J'ai entendu nos maîtres en discuter hier soir. Ils vont nous donner à des amis, ajouta Chatoune.

Ma mère Charlotte se promenait de long en large dans la pièce qui servait de chambre à coucher à tous les membres de notre club félin. Elle frôlait les murs, frottait son museau contre la porte, poussait quelques miaulements inquiets, puis, s'arrêtait et regardait patiemment ses deux soeurs discuter du dispersement futur de la communauté.

- Je comprends très bien nos maîtres, dit Charlotte, car au rythme où nous nous reproduisons, nous pourrions être une centaine ici, dans un an ou deux.

- Miaou! Miaou! Mais c'est affreux, nous ne nous reverrons jamais, pleurnichait tente Minette.

- Il faut bien se résigner, c'est là notre destin! confirma tante Chatoune. 
Même si j'étais toute petite à l'époque, je compris que malgré notre réputation d'indépendance, nous, les chats, avions beaucoup d'affection et d'attachement les uns pour les autres.

Quelques jours passèrent et la maison reçut toutes sortes de visiteurs. Nos mères les chattes craignaient désormais l'arrivée de toute personne étrangère à la maison. Chaque fois, elles s'imaginaient que ce serait le jour de la grande séparation. Même Ti-Sec, le vieux chat "marabout" n'était pas le bienvenu dans notre groupe; dans les circonstances, Minette, Chatoune et Charlotte refusaient toute hospitalité à ce vieux matou hypocrite et sans courage.

- Va-t'en! s'était écriée Chatoune en le voyant arriver. Ta place n'est pas ici!

- Tu n'es bon qu'à semer la chicane! ajouta Minette.

Elle avait raison la pauvre tante Minette. Chaque fois qu'il venait faire son tour, l'atmosphère était tendue, et souvent, on en venait aux griffes.

- Je vais te faire voir, moi! cria Ti-Sec en griffant et mordant Minette à la gorge.

C'est alors que maman Charlotte intervint. Courbant le dos comme si Ti-Sec avait été un chien errant, elle poussa quelques toussottements de félin, en guise d'avertissement.

- Je t'avertis Ti Sec, tu sors immédiatement d'ici et tu nous laisses en paix sinon tu vas le regretter! miaula Charlotte.

- Tu crois me faire peur avec tes airs de grand fauve, garde ta salive pour laver tes petits! hurla Ti-Sec, en courbant le dos lui aussi, prêt à attaquer...

Voyant que le débat s'enflammait, Blaise, le propriétaire de la maison, mit Ti-Sec dehors, regrettant même de l'avoir fait entrer. 
II avait pitié de ce vieux chat qui, de temps à autre, venait chercher pitance chez lui. Cela devait lui rappeler je ne sais quel ami, un peu clochard...

J'admirais Charlotte, ma mère. Chaque fois que je le pouvais, je tentais de l'imiter. Ainsi, peu après cet événement, j'avais appris à courber le dos devant un danger réel ou imaginé, à tousser comme une grande chatte, à montrer les dents et les griffes à qui voulait bien constater que je prenais très tôt le chemin de ma race.

Je miaulais comme une grande, je courais, sautais et me roulais sur le dos en essayant d'épater ma mère, mes tantes nourrices, mon père un peu absent, mes frères et soeurs et les propriétaires de la maison.

Parmi les visiteurs qui vinrent ce jour-là, l'un d'eux se prit d'affection pour Charlotte et moi. Durant la nuit, ma mère lui avait plusieurs fois rendu visite. La veille, il m'avait prise sur lui, $m^{\prime}$ inventant toutes sortes de noms pour savoir lequel $m^{\prime}$ irait le mieux. Chatonne, Frisette, Chavante, tous les noms y passèrent. II demeurait indécis. Pouvait-il savoir que j'avais déjà un nom?

Charlotte savait ce qui nous attendait. Elle m'avait porté une attention toute spéciale ce soir-là. Elle $\mathrm{m}$ 'avait cajolée, léchée et faite belle pour le lendemain.

Le sol habitué au soleil printanier reprenait pour quelques jours son manteau d'hiver, son poil de froid et le visiteur se demandait s'il devait ou non partir ce jour-là. Pourtant le ruisseau de la rose, gonflé, chantait un aurevoir mi-triste mi gai. Nous déjeunâmes en silence, parce qu'il n'y a rien à dire quand on part pour toujours. Puis je m'assoupis, comme pour ne pas m'enraciner à mes souvenirs. Je fis mes adieux en rêve; je crois que cela était 
mieux ainsi...

Lorsque je m'éveillai, nous étions, Charlotte et moi, dans une boîte sombre; ma mère bougeait nerveusement: elle était inquiète. On nous amenait à l'automobile qui allait nous transporter très loin... beaucoup plus loin qu'une petite chatte ne pouvait se l'imaginer.

- Miaou! Dis maman?... Où va-t-on dans cette boîte noire? Dis maman! fis-je toute étourdie d'être ballottée ainsi.

Charlotte, trop préoccupée, ne me répondit pas. Elle savait que cette boîte avec un peau d'eau, de nourriture et une couverture de laine, était notre seul "baluchat".

II était difficile de savoir combien de temps allait durer le voyage. C'est cela qui rendait Charlotte nerveuse. Durant toute la durée du trajet entre la maison et la voiture de notre nouveau maître, ma grande héroïne de mère se tint la tête sortie, pour savoir ce qui se passait... et voir la maison rapetisser tranquillement et disparaître derrière les arbres.

Le séjour dans la petite boîte fermée $\mathrm{m}$ 'apparut très long... C'était étourdissant d'être transportées ainsi. Chaque fois que I'homme s'enfonçait dans la neige, nous subissions le contrecoup. Une fois dans l'automobile, notre nouveau maître ouvrit la boîte et nous permit de circuler à notre guise: enfin de la lumière...

Le conducteur du véhicule semblait murmurer à Charlotte des mots doux. II prenait une voix plus claire pour miauler avec nous.

- As-tu peur ma belle minoune? Hein? Non non non... C'est pas dangereux... Tu va voir, tu vas aimer ça chez nous.

Et il lançait quelques sons bizarres:

- MA BELLE MANOUNI À MAKAZANOUNI! 


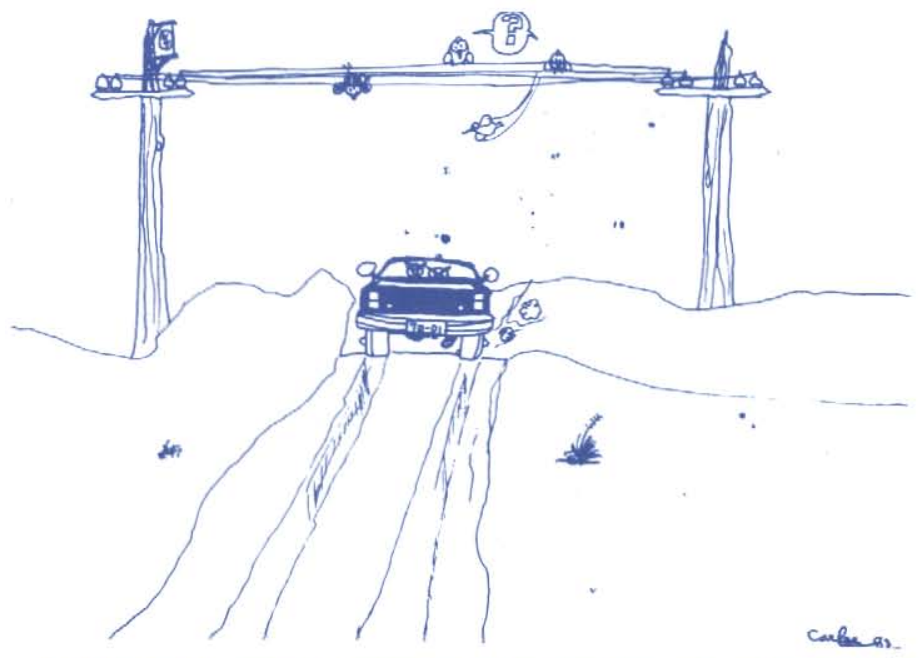

À Montréal, nous fûmes laissées seules, ma mère et moi, à l'intérieur de cette maison roulante ancrée pour quelque temps dans une rue. Je sentis que ma mère Charlotte était plus calme. Cet arrèt lui faisait beaucoup de bien. Elle s'habituait peu à peu, elle aussi, à sa nouvelle vie:

- Enfin ça ne bouge plus lança-t-elle, je devenais de plus en plus étourdie.

Mais la ville ne fut pas d'un grand attrait. II pleuvait à plein ciel et tout était sombre: beaucoup de gros chats roulants, avec des gens pressés... Nous repartîmes: tout le monde s'était bien reposé, et avait bien mangé. Nous étions maintenant cinq à bord. Deux autres humains voyageaient avec nous.

Charlotte me raconta le trajet, Saint-Hyacinthe, Drummondville, le détour par I'arrière-pays jusqu'au petit village de SaintJean-Vianney dans la région de l'amiante.

Dans la maison où nous arrêtames, beaucoup de choses me rappelaient l'endroit de ma naissance: la bonne odeur du bois qui chauffe dans la fournaise, la musique du décor dans mes oreilles de chattes, les meubles rustiques. Mais il y avait une différence... 
Deux gros chiens faisaient la pluie et le beau temps dans toutes les pièces. Ils n'étaient pas méchants: mais quand ils voulurent s'approcher de trop près, Charlotte fut encore bien plus maligne que toutes les fois où $\mathrm{Ti}-\mathrm{Sec}$ était venu troubler la tranquillité de notre petite communauté du ruisseau de la rose.

Le gros chien noir s'était approché le premier, pour venir jouer avec les nouveaux arrivés:

- N'approche pas de ma petite! cria Charlotte, sinon je te griffe le museau.

- Wouf! Wouf! c'est seulement pour jouer riait Ti-Noir.

Charlotte s'était hérissée de tous ses poils, et son dos arqué montrait qu'elle était prête à bondir, à la moindre riposte de $\mathrm{Ti}$ Noir. II s'approcha de nouveau et ma mère passa à l'attaque, les griffes sorties, la gueule lançant des menaces de fauve. Ma mère eut raison de son premier assaillant qui recula sous les coups de griffes. Je m'étais faite toute petite dans un coin. J'avais peur des cris et des jappements qui s'échappaient de la bagarre.

Gaspard, I'autre chien de la maison, n'avait rien manqué de la scène. Bien conscient qu'il perdrait la face s'il reculait devant un adversaire qui ne faisait pas le dixième de son poids, celui-ci se lança sur Charlotte qui dut céder devant I'air décidé de son énorme opposant. Tout se fit dans un tumulte de cris de chatte et de chien. J'ai bien pensé qu'il allait dévorer ma mère, tellement sa gueule était grande et ses dents pointues.

- Je vais te montrer qui est le maître ici, avait jappé Gaspard, dans son élan pour faire reculer Charlotte.

A partir de ce moment, on nous réserva une pièce fermée pour ne pas que la bagarre reprenne à nouveau. II était bien clair que ma mère ne laisserait pas les chiens m'approcher et que Gaspard ne céderait pas sa place de gardien du foyer.

Charlotte était fière de $\mathrm{m}^{\prime}$ avoir défendue. Elle me faisait la toilette en racontant ses nombreuses expériences avec les chiens. 
- Souvent, me disait-elle, les chiens et les chats se lient d'une profonde amitié quand ils sont élevés ensembles; mais il faut toujours se méfier des chiens qu'on ne connaît pas: ils ne cherchent qu'à nous courir après et à nous faire des misères.

La nuit se passa sans trop de cauchemards. La matinée fut courte, et nous fûmes vite replacées dans la voiture, maman et moi.

Nous avions déjà trouvé la tranquillité dans le changement. Non pas que nos souvenirs s'étaient effacés de notre mémoire, non pas que notre vie n'était pas dérangée par ce périple qui semblait sans fin, mais nous avions remarqué une singulière parenté entre les décors qui fuient et tous les objets animés qui nous font rêver le jeu... De la balle de laine à la souris, d'un pied humain à une poitrine qui se gonfle et se dégonfle, et fait du vent chaud. Nos jeux concrets devinrent des jeux dans l'imaginaire.

- Montmagny! quelques chats gris! s'amusa ma mère.

- Sapristi! le ciel est gris pour Mistigris! répliquai-je en faisant l'effort savant de quelques rimes poilues.

- Vas-y! Vas-y! renchérit Charlotte.

- St-Jean Port Joli! que tu me dis! une minette sur un porc joli! Youpie! miaulai-je une fois de plus.

La pluie, encore une fois revint après la neige, comme le soir bruni après un jour très clair. Rivière-du-Loup...

- C'est comme de l'eau pour les chiens. De loin je ne crains riens! ricana ma mère.

- C'est comme de l'eau pour les chiens! Ça fait rien! balbutiai-je sans comprendre.

- Une autre fois, compte les miaulements sur tes pas! ordonna ma mère. 
- Mes pas! Mes pas! fis-je avec l'ironie des petits sires de gouttière.

Rimouski, ses clochers et son fleuve large comme une mer, nous valurent une longue attente. Petit à petit les fenêtres se couvrirent d'un gros manteau d'hiver. La rue ronronnait doucement sa dernière tempête inattendue. route.

Nous passâmes la nuit dans un petit appartement bordant la

Le lendemain était une neige lourde. Je ne comprenais plus le voyage. J'avais encore changé de maison et passé la nuit sans ma Charlotte aux huit tétines accueillantes. Ma mère, laissée dans la voiture, j'avais dû pour la première fois composer ma propre chaleur et dormir au seul poil de mon égarement.

La porte du matin s'ouvrait sans le déjeuner chaud et maternel. Lorsque je pus enfin me délecter sous le ventre chaud de Charlotte, le soleil me fit un grand sourire, et mes yeux s'affolèrent sous la gigue de mes moustaches avares d'inconnu et d'émerveillement aux petits signes familiers.

La grosse maison brune avait franchi ses derniers espaces dans le beau temps revenu: le bout du voyage! Le pays du futur et du ronronnement dans les espaces printaniers. Nous serions les tigres domestiques de cette nouvelle demeure: nouveau territoire dont Charlotte fixerait les alentours.

La maison est grande, immense, fatigante. Elle nous a déjà un peu et nous façonne de ses exigences. Dans les murs on entend gratter les souris qui seront notre pitance.

Le terrain d'environ trois âcres et la maison de trois âges me font un univers immense. Le coucher de soleil sur le fleuve fait un phare avec ses bâteaux jouets qui remontent la nuit jaune, orange et puis mauve jusqu'à la noirceur qui me rend les yeux en amandes, aux clins d'oeil des aurores boréales.

- De gros chats qui clignent de l'oeil à travers la nuit! 
La porte de cave n'a pas d'écrou. L'humidité et la fraîcheur un peu moisie grimpent les marches jusqu'au premier dans la cuisine.

En quelques jours, j'ai appris moi aussi à monter l'escalier, à appeler mon maître pour qu'il me laisse gambader à l'aise dans la maison. II suffit de gratter avec mes griffes dans la porte et il vient. C'est fou ce qu'il s'est vite apprivoisé.

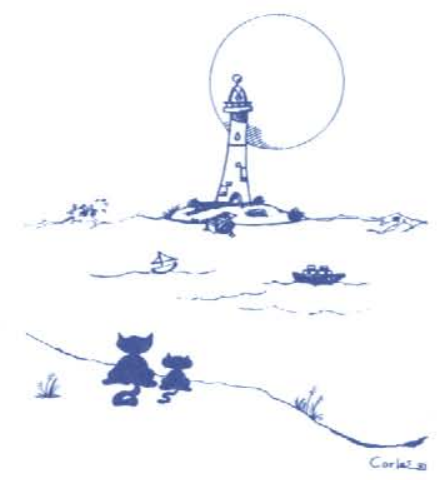

La grange au du foın plein la gueule et l'étable inhabitée meugle son ennui. Le jardin est encore en neige.

II fait très beau près du poulailler qui baille aux nuages. Les rouleaux de broches qui traînent sont des toiles d'araignées.

Le toit du deuxième étage est bas et courbé: c'est un vieillard qui guide et conseille toute la maisonnée.

Je ne suis pas encore allée aux champs. Ma mère m'a dit qu'il y a de gros tas de roches qui ramassent les vieux souvenirs.

Tout nous marque en dedans. Cet été, mon enfance grandira. Je jouerai aux champs et je vivrai mon beau rêve dans un langage qui $\mathrm{m}^{\prime} \mathrm{embrasse}$ comme un univers:

- Ma belle Ma Nou Ni A Ma Ka Za Nou Ni!

\section{F I N}

